Musées, Patrimoine et Culture scientifiques et techniques

$116 \mid 2008$

mars - avril 2008

\title{
Un musée dans une usine
}

\section{Manar Hammad}

URL : http://journals.openedition.org/ocim/369

DOI : 10.4000/ocim.369

ISSN : 2108-646X

Éditeur

OCIM

Édition imprimée

Date de publication : 1 mars 2008

Pagination : 10-20

ISSN : 0994-1908

Référence électronique

Manar Hammad, «Un musée dans une usine », La Lettre de l'OCIM [En ligne], 116 | 2008, mis en ligne le 01 janvier 2011, consulté le 19 avril 2019. URL : http://journals.openedition.org/ocim/369 ; DOI

10.4000/ocim.369

Tous droits réservés 


\section{Un musée dans une usine}

\section{Manar Hammad *}

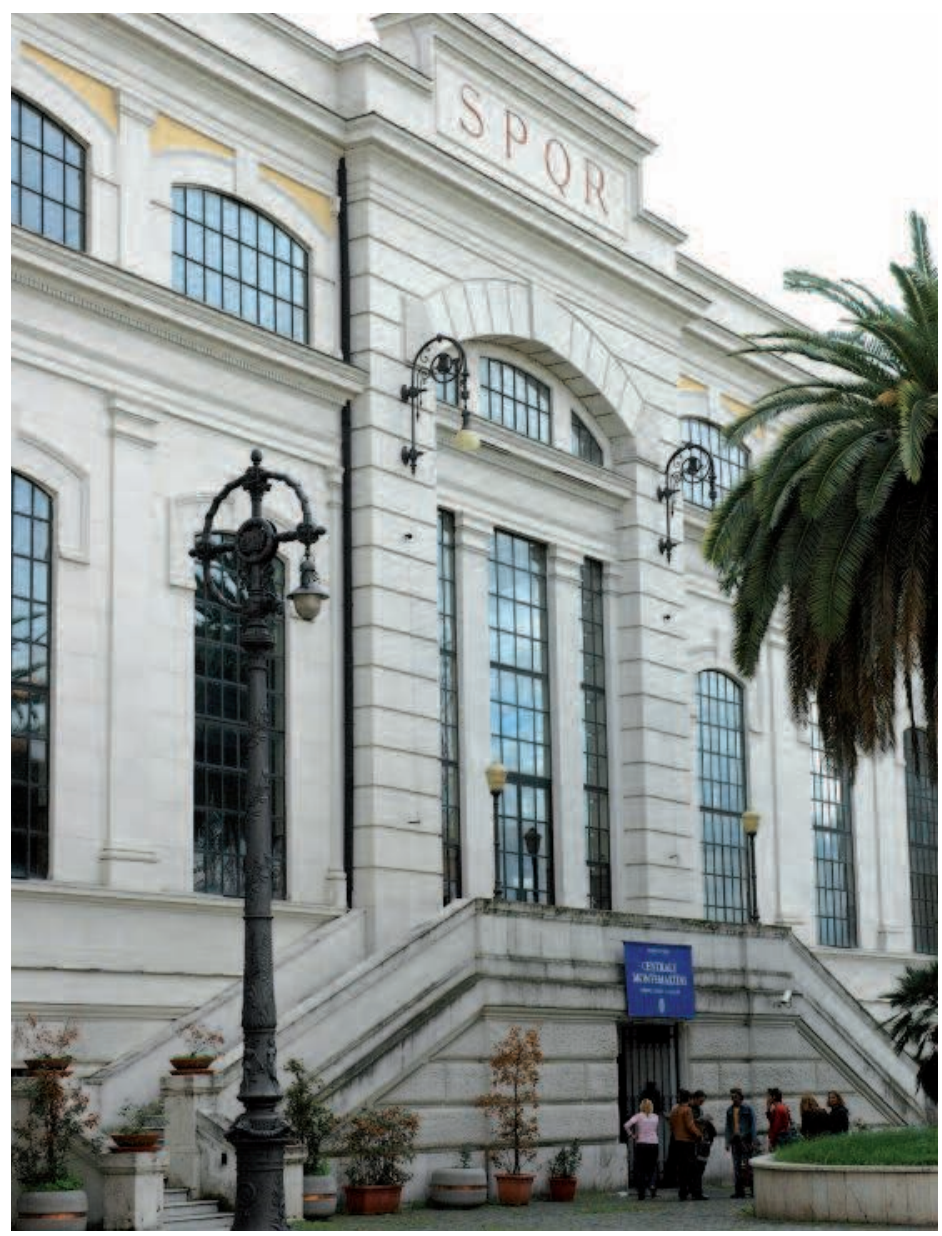

Entrée du musée de la Centrale Montemartini (c) M. Hammad

* Manar Hammad est architecte DPLG et docteur en sémiotique manar@noos.fr
Le musée de la Centrale Montemartini à Rome présente des œuvres antiques dans un cadre industriel. Conçu comme un lieu d'accueil temporaire pour les œuvres évacuées des musées capitolins pendant les travaux d'aménagement du Palais Caffarelli, il a été l'occasion de tester des idées muséographiques qui seraient à mettre en œuvre sur le site capitolin lors du retour des œuvres. Il est ici soumis à une analyse sémiotique.

\section{Le musée de la Centrale Montemartini (1)}

L'implantation de la Centrale Montemartini a été déterminée par des impératifs industriels, en fonction d'avantages technico-économiques liés à la proximité du Tibre. Néanmoins, l'architecture témoigne d'un souci monumental : la façade est dessinée avec soin, un escalier extérieur à double volée mène vers un étage noble. À l'intérieur, les volumes amples sont lumineux, offrant de grandes surfaces libres sous un plafond tripartite évoquant certains édifices religieux. Les éléments de béton sont dessinés avec soin et le bâtiment est signé par l'entreprise constructrice. Ces caractères témoignent du fait que le bâtiment était valorisé dès sa conception : c'est un monument de l'industrie. C'est à ce titre qu'il a été restauré et transformé en musée industriel, avant qu'on n'y ait placé des sculptures. Il en découle que l'architecture de ces lieux est indépendante de tout message formulé par les musées capitolins. Lors de l'introduction d'une collection de sculptures, un 
nouveau discours s'est superposé à l'ancien. Les machines du musée industriel ont été conservées à leur place et les sculptures antiques sont venues s'installer autour, ajoutant une collection à une autre et plaçant un discours à côté de l'autre. En termes quantitatifs, il y a plus de sculptures que de machines. En termes qualitatifs, la communication autour du musée est faite par les musées capitolins, pour la collection de sculptures. De ce fait, le visiteur de la Centrale Montemartini se déplace plus pour l'un de ces discours que pour l'autre, et le discours du musée archéologique est dominant par rapport au discours du musée industriel.

\section{La séquence des salles muséales}

et ses effets de sens

Les contraintes de base à la Centrale Montemartini sont architecturales : il y a deux corps de bâtiment accolés ( $\mathrm{M}$ et $\mathrm{C})$, chaque corps est à deux niveaux $(0$ et 1). L'accès par le rez-de-chaussée du bâtiment $M$ distingue celui-ci. Le visiteur est orienté par les gardiens vers la salle $\mathrm{C} 0$ où un parcours en $\mathrm{U}$ le ramène au point de départ. De la salle M0, un escalier

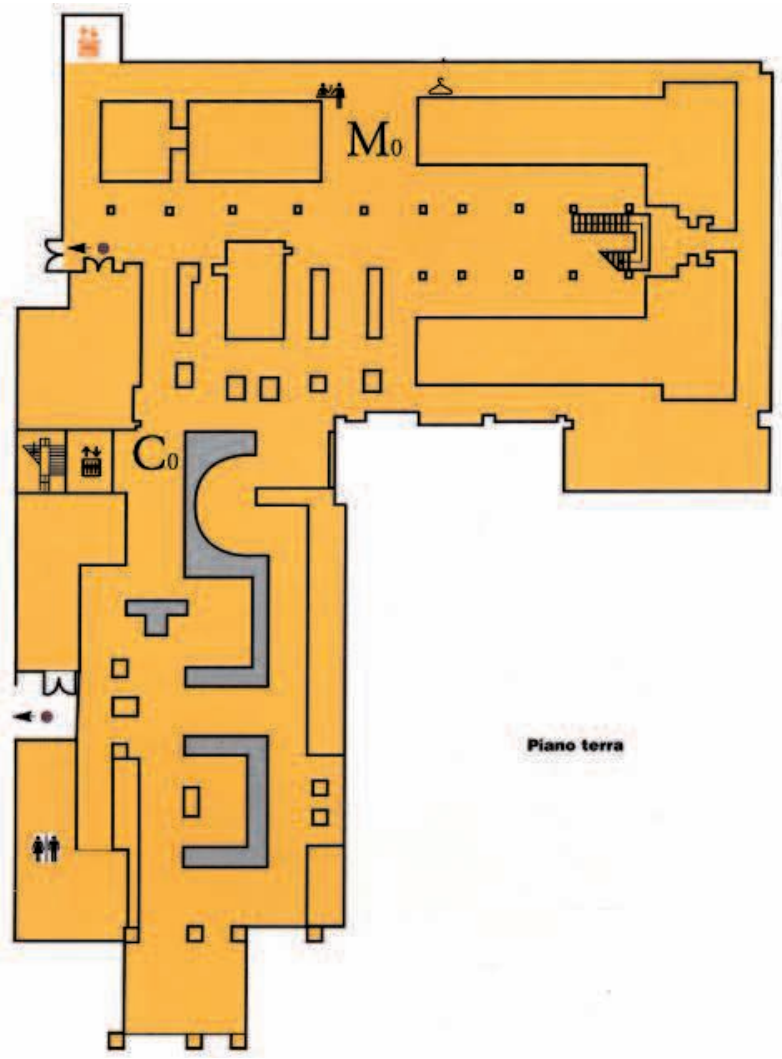

Plan du rez-de-chaussée du musée avec les salles $M O$ et $C O$ conduit à vers la salle $\mathrm{M} 1$, où une certaine liberté de parcours est loisible. Une porte fait communiquer la salle M1 avec la salle C1, où le parcours est libre. Pour sortir, il faut repasser par Ml puis par M0.

Les lieux sont articulés par quelques oppositions perceptives : le rez-de-chaussée est sombre alors que l'étage est lumineux, il est bas de plafond alors que l'étage possède des plafonds hauts surhaussés par une nef centrale. Au rez-de-chaussée, les cloisons sont proches du visiteur, alors qu'à l'étage elles sont éloignées. Le passage du rez-de-chaussée à l'étage oppose un espace comprimé et sombre à un espace élargi et lumineux : à la tension succède la détente. À ces oppositions spatiales correspondent des oppositions entre les univers des œuvres montrées : les objets de C0 (caractérisée par la tension) renvoient aux origines de Rome, ceux de Ml et Cl (caractérisées par l'expansion) renvoient à l'épanouissement impérial. L'aménagement des salles muséales qualifie les univers antiques auxquels renvoient les collections.

Les noms des salles muséales sont en décalage par rapport aux contenus. La salle C0 réunit des objets datant des origines de Rome, elle est dénommée

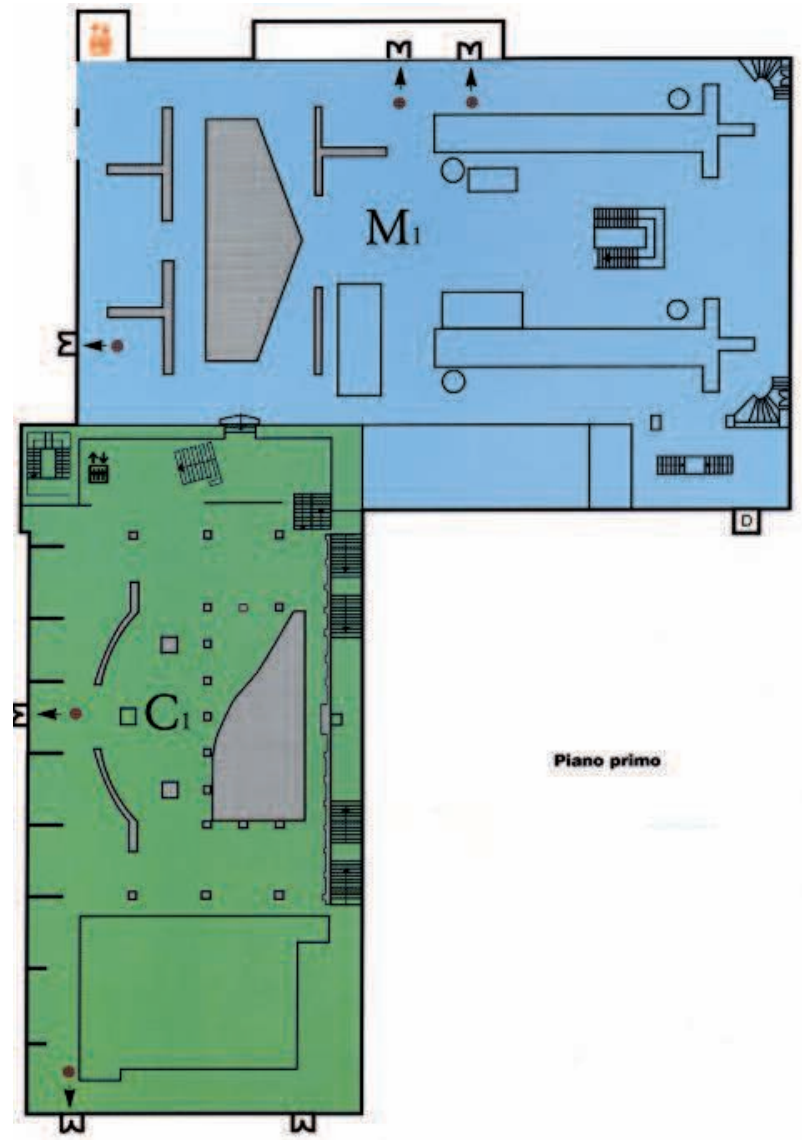

Plan du $1^{\text {er }}$ étage du musée avec les salles $M 1$ et $\mathrm{Cl}$ 
Salle des Colonnes. La salle M1 réunit des œuvres provenant du centre impérial de Rome, elle est dénommée Salle des Machines. La salle Cl réunit des œuvres provenant des jardins gentilices, elle est dénommée Salle des Chaudières. Le maintien des dénominations techniques antérieures aux salles muséales produit trois effets de sens : le musée industriel est affirmé face à l'omniprésence des sculptures antiques; la présence des objets antiques ne serait que temporaire ; la perception du visiteur amateur de sculptures est brouillée, retardant la saisie de la logique du musée archéologique.

\section{Les lieux de la séquence muséale}

\section{La crypte des origines $\mathrm{CO}$}

Les objets de la Salle des Colonnes C0 sont au croisement d'un critère historique (antérieurs à l'Empire : périodes archaïque et républicaine) avec un critère topographique (périphérie de Rome à l'époque considérée : Forum Boarium et nécropoles). Provenant d’un sol qui fut hors de la ville de Rome, ces objets comptent parmi les plus anciens que l'on connaisse de la ville (2). Ce qui signifie que le savoir que nous en tirons sur les origines de Rome ne provient pas de ce qui en fut le centre mais de sa périphérie ancienne.

Au plafond subsistent des traces de l'ancienne usine : des trémies pyramidales, des trappes coulissantes, des poulies et des pignons, peints en noir sur fond blanc, témoignant du fait que l'on recevait à cet étage les cendres de combustion des chaudières du dessus. Les murs de la salle sont recouverts de panneaux peints. Le matériau (bois aggloméré) serait indifférent s'il ne témoignait d'un souci d'économie. Sont ainsi occultées les colonnes qui ont donné leur nom à la salle, produisant la perplexité du visiteur. La couleur des murs est d'un jaune clair, correspondant, en valeur affaiblie, à la couleur dominante des pierres. Ici, la forme est plus sombre que le fond. Aucune lumière naturelle n'est admise dans ce local, lui donnant le caractère de crypte des origines. Toutes les sources de lumière sont directionnelles, aucune lumière d'ambiance n'est fournie : la valeur déictique de l'éclairage est impérative et l'on ne voit que ce qui est éclairé.

\section{La crypte technique $\mathrm{MO}$}

La salle est allongée, son plafond bas porté par de courtes colonnes. Les murs sont blancs, les machines exposées noires. Une seule exception fait contrepoint : une Aphrodite de marbre blanc se détache devant la masse noire d'un condensateur. Dans l'univers visuel du musée, les autres sens sont habituellement neutralisés : il est interdit de toucher, et l'on invite au silence. Il n'est question ni de goûter ni de sentir une odeur. Or, dans cette salle M0, une odeur surprend : celle de l'huile de moteurs. Dégagée par les machines présentes, elle caractérise ce lieu et contribue à son identité. Par son étrangeté, elle crée un effet d'ailleurs : le visiteur n'est plus dans un musée d'antiquités, mais dans un environnement industriel.

Les masses de deux moteurs dominent cet espace. Deux centrifugeuses sont reconnaissables à proximité d'un condensateur. Quelques outils de grande taille sont disposés à côté. Un unique câble électrique témoigne qu'en ces lieux, cette mécanique visait à produire de l'électricité. Il met en évidence un aspect paradoxal du musée industriel : dans cette ancienne centrale électrique, l'électricité est invisible. Tout ce qui est montré est en amont de l'électricité : des moteurs, des chaudières, des turbines, soit le dispositif industriel transformant une forme de l'énergie en une autre forme de l'énergie. De ce fait, l'énergie même, en tant qu'entité abstraite, est placée au cœur du musée industriel de la Centrale Montemartini.

\section{La Salle des Machines M1}

Le passage par l'escalier intérieur assure la liaison entre deux lieux d'exposition sans faire passer par un espace tiers. Le passage à travers une trémie horizontale renforce les contrastes : par sa relative rareté, il s'oppose au passage ordinaire des portes verticales. Le passage du bas vers le haut se double d'une transition rapide du sombre vers le lumineux, du condensé vers l'expansé. Sans énoncé verbal, les qualités physiques des lieux sont projetées sur le contenu des salles : aux débuts sombres de la Rome

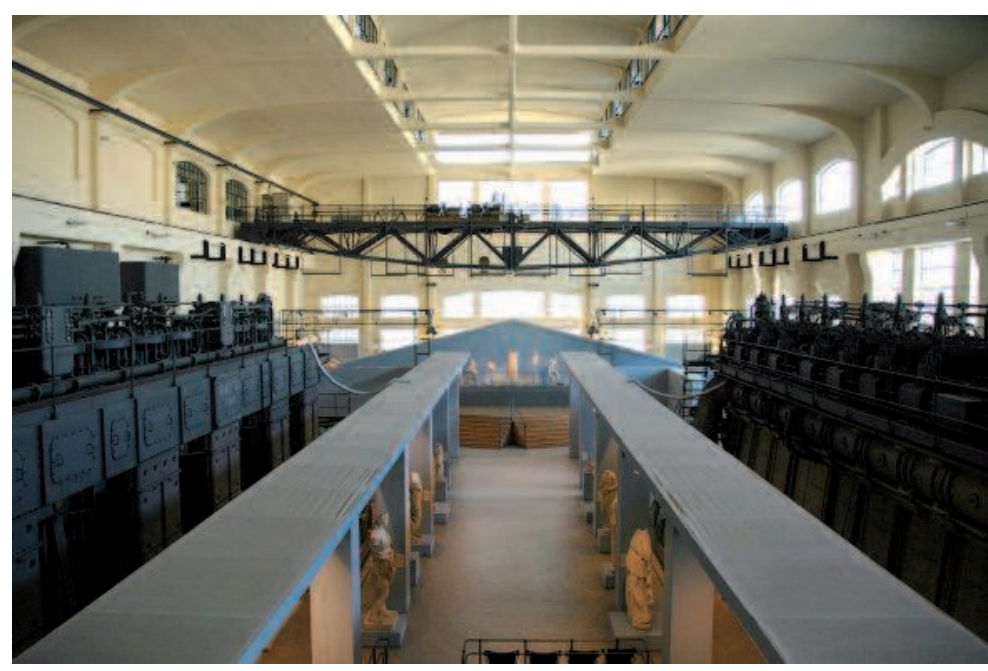

La Salle des Machines avec au plafond la nef surhaussée et au sol les portiques et moteurs du dispositif basilical ○ M. Hammad 
républicaine succède le développement lumineux de la Rome impériale, aux mobiliers et figurations funéraires succèdent les représentations divines, à la crypte chtonienne succède l'étage céleste.

$\mathrm{Au}$ plafond, une bande centrale longitudinale est surélevée sur des poutres transversales, ménageant une nef axiale aérienne dotée de deux rangées de fenêtres qui assurent un éclairage zénithal dans un volume libre de toute colonne intermédiaire. Cet aménagement était déjà celui de la centrale électrique. Il reprend l'un des dispositifs de l'architecture basilicale qui caractérise les édifices de prestige depuis l'Âge du Bronze. Par cet aménagement, les constructeurs donnent au bâtiment une dignité certaine : pour eux comme pour les commanditaires, ce n'était pas un simple hangar abritant des machines, c'était un palais de l'industrie. Sous le plafond basilical, le musée archéologique a aligné des piliers réunis par des poutres formant architrave, adoptant au sol une disposition basilicale selon l'axe majeur de la salle. En bref, l'aménagement muséal assume le parti architectural antérieur. Il y a, en ce lieu, un accord total entre les dispositifs spatiaux du musée technique et du musée archéologique. L'organisation basilicale de la salle occupe les deux tiers du grand axe. Dans le tiers restant, une plateforme transversale barre l'espace, réalisant trois opérations simultanément : elle surélève par rapport à la salle le groupe de l'amazonomachie, lui attribuant un statut supérieur au reste ; elle ménage sur son arrière des lieux semi-fermés pour des objets ; elle coupe la Salle des Machines à la hauteur de la porte mettant cette dernière en relation avec la Salle des Chaudières, facilitant l'identification de la suite de la séquence muséale.

Les objets proviennent de fouilles effectuées en deux périodes. La première a suivi linstallation de la capitale de l'Italie à Rome en 1870. La seconde correspond aux travaux d'embellissement entrepris durant les années 1920-1930. Les objets du musée, qui étaient dotés de deux repères historiques (celui de la création, celui de la mise en œuvre à Rome), se trouvent dotés d'un troisième repère temporel : celui de la découverte. Un quatrième moment, celui de linstallation du musée, représente une phase déterminante dans la constitution du sens, de sa sédimentation historique et de son accumulation dans l'objet.

\section{La Salle des Chaudières $\mathrm{Cl}$}

Une grande baie, ouverte dans le mur séparant M1 de $\mathrm{Cl}$, assure le passage. Opposée à la trémie horizontale, cette ouverture verticale réunit les salles de l'étage pour les opposer ensemble aux salles du rezde-chaussée. La baie s'ouvre dans le prolongement de la plateforme portant l'amazonomachie de Ml. Placé devant celle-ci, le visiteur entrevoit dans C1 un groupe de marbre figurant un homme grimpant sur un char tiré par un cheval (Thésée enlevant Antiope). L'un des montants de la baie cache partiellement le cheval. La mise en visibilité partielle produit sur le visiteur un effet d'attraction : il se rapproche pour tout voir. Dans ce dispositif spatial, nous reconnaissons une manipulation non verbale du visiteur, le dotant de la modalité du vouloir.

Au plafond, la Salle C1 présente un dispositif d'éclairage basilical, identique à celui de la Salle M1. Il y eut trois chaudières dans cette salle. Lors du réaménagement en musée technique, deux chaudières furent démontées. La masse de celle qui subsiste occupe près du quart du volume de la salle. Les circulations ménagées autour d'elle font que son emprise au sol avoisine le tiers de la surface. Elle détermine l'espace d'exposition par soustraction : les antiquités occupent ce qui reste de la salle. Dans la portion de salle laissée par la chaudière, une grande mosaïque occupe le côté oriental, bloquant le tiers de l'espace résiduel. Elle effectue la deuxième opération soustractive sur la salle, déterminant l'organisation du reste le long d'un axe transversal : de la passerelle dominant la mosaïque, le visiteur identifie l'axe mineur de la salle, sur lequel une exèdre arrondie a été dressée, ses parois pleines s'opposant aux portiques.

Il résulte des opérations soustractives sur la salle, de la non-utilisation de l'axe longitudinal du plafond, et de l'utilisation d'un axe transversal décentré, que la Salle des Chaudières ne dégage pas une impression

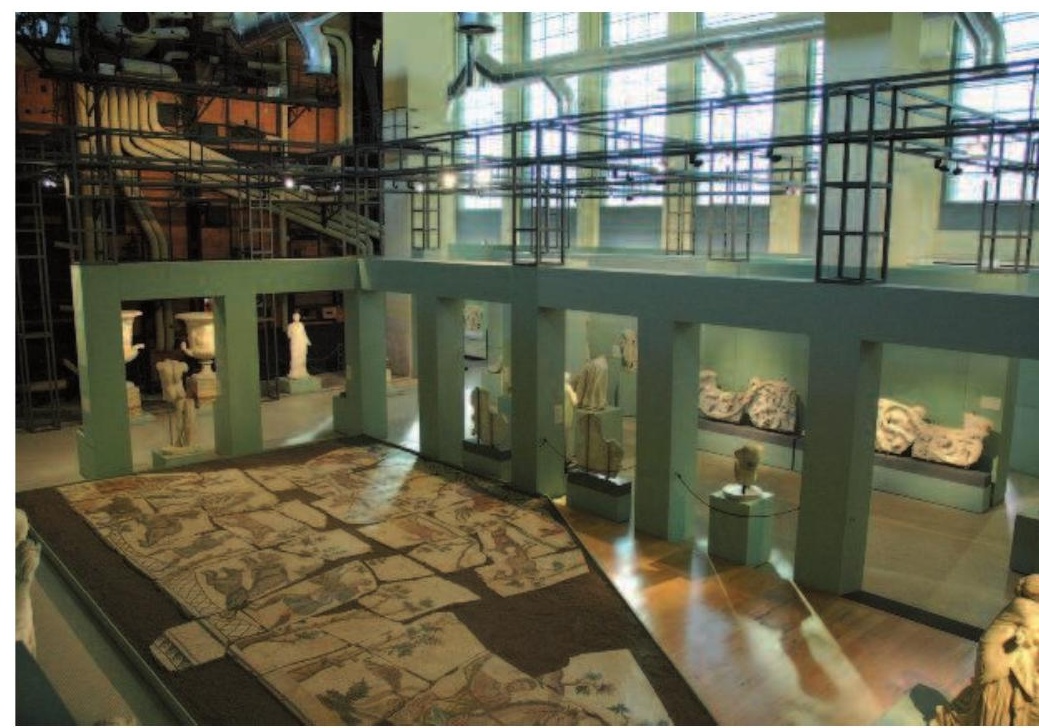

La Salle des Chaudière avec au premier plan un mosaïque d'époque antique et à gauche la masse de la chaudière méridionale (๑) M. Hammad 
d'ordre. Non pas qu'elle soit désordonnée, mais elle n'est pas clairement ordonnée. Opposée à la Salle des Machines, où l'ordre basilical était clair, nous avons ici moins d'ordre.

La totalité des objets exposés dans cette salle provient des campagnes de fouilles consécutives à 1870, sur les lieux destinés aux ministères et à leur personnel, hors la ville de l'époque. Cela correspond à ce qui fut la périphérie de la Rome impériale, où dès la fin de la République les familles gentilices se firent aménager de grandes demeures dans la verdure. Les fouilles trouvèrent de nombreuses sculptures groupées : elles étaient encore sur le site où elles avaient été disposées par leur propriétaire. Certaines étaient encore dans les niches murales qui les accueillaient à l'époque antique. D’autres, retrouvées au sol devant une niche, étaient tombées de celle-ci. De ce fait archéologique, les conservateurs ont tiré un concept muséographique : remettre ensemble, dans le musée, les pièces trouvées groupées in situ. Ainsi, le programme muséal de cette salle ne se construit pas à partir des objets mêmes qui y sont réunis, mais à partir des conditions de leur exposition antique. En restituant une ancienne disposition, le musée replace le visiteur dans la situation du spectateur antique vis-à-vis de ces pièces. En termes sémiotiques, l'énonciataire est invité à effectuer un débrayage et à adopter la vision d'un autre sujet. On comprend dès lors la distribution spatiale et la couleur de l'aménagement dans cette salle : l'exèdre, les piliers, les petits volumes visent à reproduire l'ambiance spatiale des jardins dans lesquels étaient disposées les sculptures ; la couleur verte évoque celle des jardins. L'aménagement muséal transcrit dans son expression une manière antique de mise en espace, correspondant à une manière de regarder l'art.

\section{Le discours urbain de la muséographie à la Centrale Montemartini}

La Centrale Montemartini ne tient pas un discours chronologique : le temps y est apparu lié à l'espace, ces deux dimensions étant imbriquées, imposant de différencier un temps de l'énoncé inscrit dans les objets et un temps de l'énonciation inscrit dans les fouilles. Le temps de l'énoncé s'organise en avant Auguste/après Auguste, alors que le temps de l'énonciation s'organise en fouilles de 1870/fouilles de 1920. L'espace s'organise selon l'opposition centre/périphérie, tant pour l'énoncé que pour l'énonciation. En résumé, ce musée parle du développement territorial et culturel de la ville de Rome en redistribuant des œuvres d'art antiques trouvées dans le sol de Rome.

\section{Les discours juxtaposés des musées} archéologique et technique

Ce musée a installé la collection antique dans un bâtiment qui n’a pas été vidé de son contenu initial : il montre encore certaines de ses machines. Avant d'accueillir la collection venue des musées du Capitole, le bâtiment fonctionnait déjà comme musée technique, accueillant des manifestations culturelles. Il convient donc de l'analyser sous cet angle.

\section{Le musée technique}

\section{de la Centrale Montemartini}

Les bâtiments recevant le musée méritent l'attention : la façade a reçu, avec le perron, les pilastres et les corniches un traitement architectural monumental. Leur ossature de béton est soignée. Les volumes de l'étage sont amples, le dispositif basilical de la toiture leur donne une monumentalité certaine. Le sol des salles de l'étage est revêtu de céramiques dessinant un cadre autour des socles des machines et des murs. Au titre de sa seule architecture, le bâtiment mérite la visite.

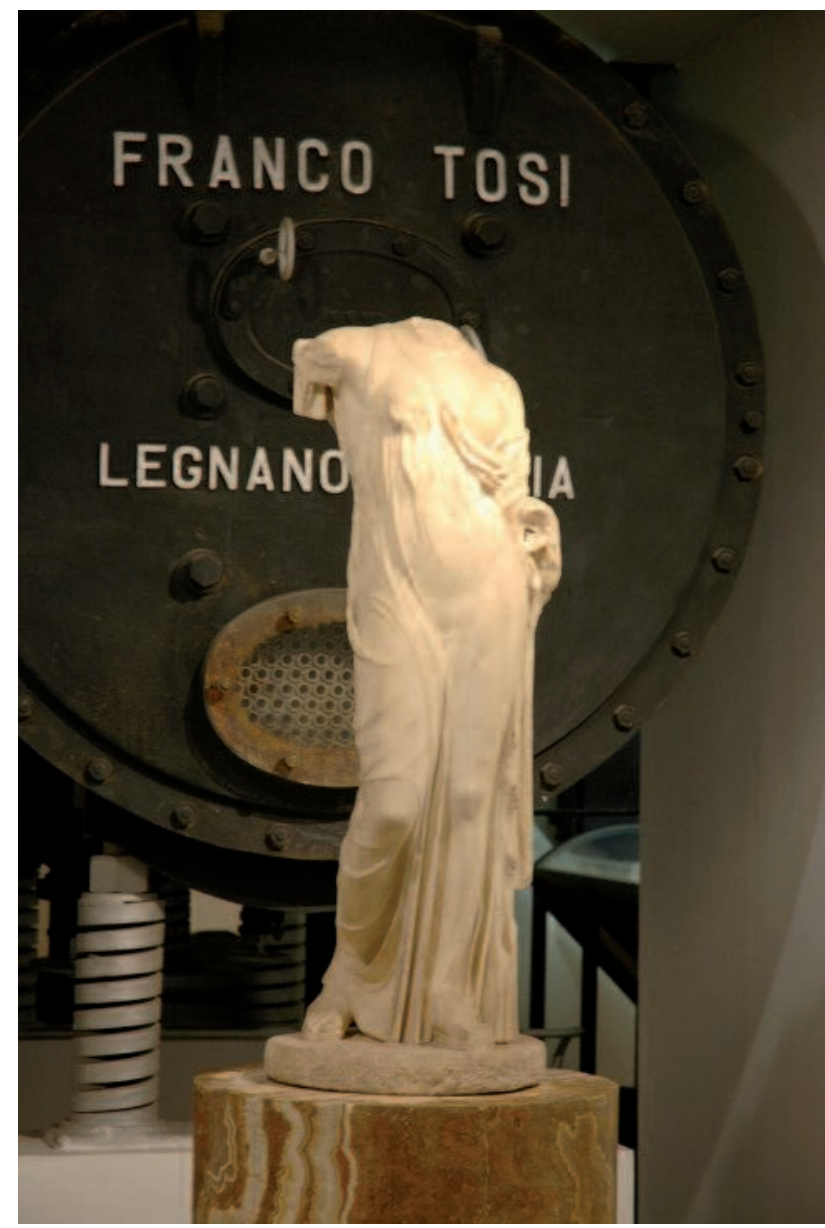

La statue d'Aphrodite placée devant un condenseur accueille le visiteur. (c) M. Hammad 
Quelques machines sont restées en place, traitées pour être montrées. Elles ont gardé leur disposition fonctionnelle. D’autres machines ont été déplacées afin d'être rendues plus visibles ou de libérer l'espace à d'autres usages. Enfin, une troisième catégorie de machines a été enlevée. On en connaît l'existence passée par des traces subsistant au sol ou par les photographies et dessins montrés dans la salle M0. Du point de vue sémiotique, une machine qui ne tourne plus et qui est montrée dans un musée est un objet qui a connu un processus de désémantisation-resémantisation. Elle a été extraite de la chaîne productive qui lui donnait un sens, pour être insérée dans une chaîne muséale qui lui donne un autre sens. Ses capacités productrices sont suspendues. Privées de leurs capacités pragmatiques, ces machines sont restreintes à leurs capacités cognitives et esthétiques.

Peu d'aménagements muséaux sont attribuables au musée technique. Au rez-de-chaussée, quelques panneaux documentaires sont placés entre les moteurs. Ils montrent des plans, des dessins fonctionnels, des photographies. Cependant, le caractère technique de certains dessins les rend incompréhensibles à beaucoup de visiteurs : ils présupposent un lecteur disposant d'un bagage technique. À l'étage, l'un des moteurs de la Salle des Machines laisse voir, à travers un panneau transparent, une bielle sur vilebrequin. Ce lieu du regard dans le ventre de la machine est à peine indiqué : il ne se lit que par un intervalle large interrompant le rythme régulier des socles portant des têtes de marbre. Dans la Salle des Chaudières, la chaudière subsistante laisse entrevoir son intérieur par deux ouvertures ayant la taille d'une porte.

En résumé, les aménagements muséaux de la Centrale électrique sont relativement discrets, et les machines sont censées parler pour elles-mêmes. Aucune tentative n'a été faite pour donner une valeur plus générale au musée technique en y retraçant l'histoire de la production électrique, ni celle des moteurs et des chaudières. Il se limite à ses monuments propres et s'adresse à un visiteur possédant déjà une culture technique.

\section{Le musée archéologique}

\section{de la Centrale Montemartini}

Le musée archéologique regroupe des objets ayant deux caractères essentiels : ils remontent à l'Antiquité, ils ont été trouvés dans la ville de Rome. Si l'on n'est pas intéressé par la sculpture, par l'Antiquité, ou par Rome, ce n'est pas la peine d'entrer car les objets n'auraient pas de valeur. D'un point de vue sémiotique, cela veut dire que ce discours muséal ancre son énoncé dans le temps (l'antiquité) et dans l'espace (ville de Rome), présupposant un point de vue esthétique chez l'énonciataire.

\section{Les opérations communes aux musées technologique et archéologique}

- Suspendre la valeur d'échange. Qu'ils soient antiques ou récents, statiques ou mécaniques, les objets de la Centrale Montemartini ont en commun d'avoir été retirés du circuit marchand. En position statique par rapport à un environnement dynamique, ils resteront ce qu'ils sont aujourd'hui. Ce qui impose de les conserver à l'identique, et de les préserver du vol éventuel.

- Suspendre la valeur d'usage. Les objets antiques et les machines récentes de la Centrale Montemartini ont en commun de ne plus fonctionner de manière efficace : les divinités ne reçoivent plus de culte, les empereurs ne commandent plus, les sarcophages et les urnes n'abritent plus les restes des morts, les moteurs ne tournent plus, les chaudières ne produisent plus de vapeur. En bref, les objets n'ont plus de valeur d'usage. Ils ont été retirés du circuit d'activité qui leur donnait leur valeur.

- Mettre en place la valeur esthétique. En organisant l'espace pour montrer les machines et les sculptures, le musée les expose au regard. Lorsque les objets sont éclairés, mis en contraste par rapport à leur environnement, écartés les uns des autres, ils sont présentés comme des objets plastiques, sollicitant un regard esthétique.

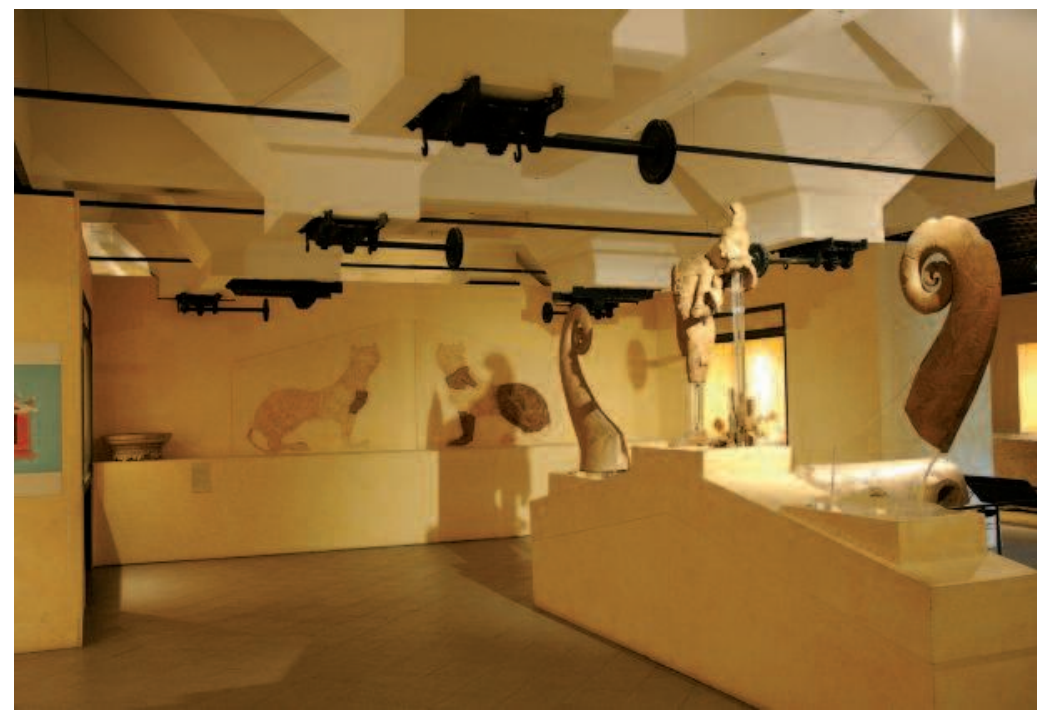

Acrotères et fronton du sanctuaire de Matris Matutæ (aire de S. Omobono, Forum Boarium) : à noter les trémies subsistant au plafond de la salle. (c) M. Hammad 
- Mettre en place la valeur cognitive. En accompagnant les objets de cartels qui les identifient, en précisent la date de création, de mise en place et de trouvaille, en repèrent le lieu de trouvaille, le musée les propose à une saisie cognitive. Par la réunion des objets en un même lieu, le musée fait apparaître des similitudes et des différences productrices de savoir : la collection est, en elle-même, un outil producteur de connaissances.

- Transformations de la valeur sémantique. En décrivant les opérations qui privent l'objet d'une valeur sémantique pour y investir une autre, nous explicitons le fait que les objets du monde naturel reçoivent, de leur composition avec d'autres objets et de leur mise en relation avec des sujets, des valeurs sémantiques qui y sont investies. Ces valeurs, qui pouvaient apparaître comme intrinsèques de prime abord, sont en fait " extrinsèques » et dérivent des opérations syntaxiques dans lesquelles sont insérés les objets.

\section{Les connecteurs d'isotopie}

Si les musées technique et archéologique cohabitent bien à la Centrale Montemartini, c'est qu'ils ont en commun quelque chose qui les relie. Les mécanismes de désémantisation-resémantisation font partie de ce noyau commun. D'autres faits sémantiques les relient, que nous appelons connecteurs d'isotopie.

- Connecteur modal: sans entrer dans le détail formel des concepts, il suffit de dire que la suspension de la valeur d'usage (ou de la valeur d'échange) équivaut à la perte de la modalité du " pouvoir fonctionner » (ou échanger). En termes figuratifs, les objets du musée, tant les dieux que les machines, sont rendus impuissants. La puissance des divinités était aussi invisible que l'est l'électricité de nos machines.

- Connecteurs figuratifs in præsentia : si les sculptures et les machines sont des objets esthétiques, elles le doivent à leurs formes, dites plastiques.

- Connecteur figuratif in situ : tant les objets archéologiques que les machines électriques se rapportent à la ville de Rome. L'espace de référence commun fonctionne comme connecteur d'isotopie entre le technique et l'archéologique, ou comme marque d'embrayage spatial entre le lieu du sujet et le lieu de l'objet.

- Connecteur figuratif universel : dans la mesure où la muséographie de la Centrale Montemartini vise à transmettre une fraction de la culture antique, elle participe au vaste programme de base des musées, celui de reconstituer les cultures disparues. Le musée technique de la centrale relève du même projet général, même s'il se positionne sur une isotopie technologique et dans une temporalité récente : il reconstitue une partie de la culture gommée par l'évolution rapide de nos sociétés.

\section{Le paradigme des musées installés dans des lieux préexistants}

La Centrale Montemartini est peut-être l'unique musée archéologique installé dans une centrale électrique conservant une partie de son matériel d'origine. Cependant, ce n'est pas le seul musée d'art à être installé dans un bâtiment industriel. De ce point de vue, elle relève d'un paradigme, celui des musées utilisant un bâtiment de récupération. À Rome même, les musées capitolins et le musée national Archéologique occupent des lieux non construits à leur intention : le Palais des Conservateurs, le Palais Caffarelli, le Palais Altemps, les thermes de Dioclétien et le Collège des Jésuites étaient destinés à d'autres usages. Leur réutilisation en tant que musées implique une désémantisation (l'usage premier est suspendu), suivie d'une resémantisation exprimée par le traitement reçu, les dispositifs de sécurité ajoutés... En d'autres termes, ces bâtiments réutilisés ont subi le traitement que subissent les objets muséaux mêmes, ils relèvent aussi de la classe des objets de musée.

Le musée de la Centrale Montemartini apparaît comme novateur dans sa muséographie et dans la relation établie entre les œuvres et leur environnement. À ce titre, il mérite une mention spéciale. Profitant de la possibilité d'expérimentation ouverte par le caractère temporaire prévu pour l'installation, il essaie de nouvelles manières de combiner des procédés disponibles dans la pratique muséale, introduisant des changements dans la continuité.

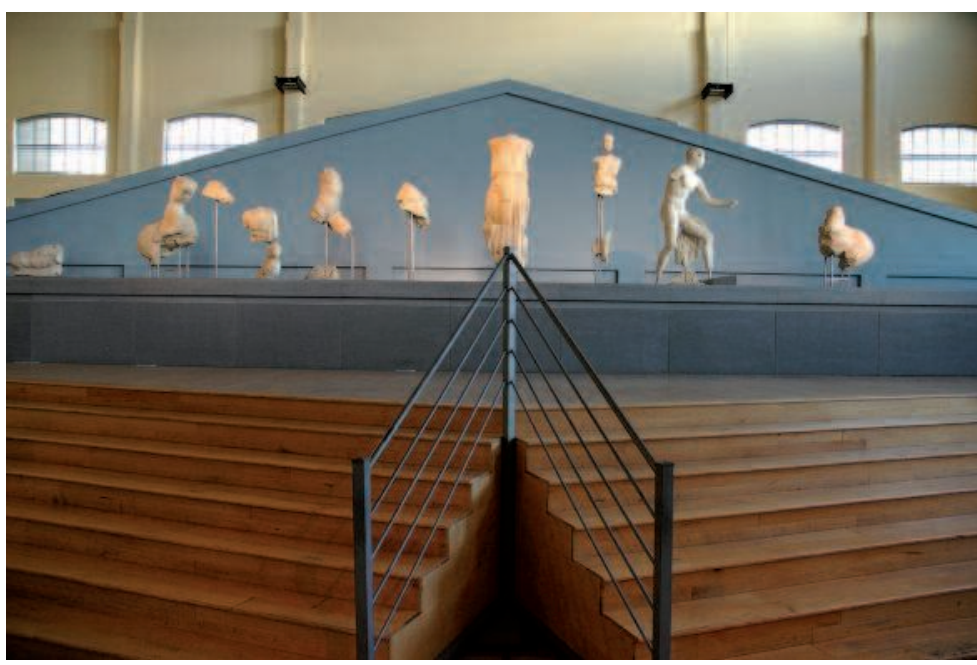

Les neuf sculptures situées sur la plateforme forment une Amazonomachie. () M. Hammad 


\section{L'isotopie dominante}

La notion d'effet de sens dominant est due au linguiste Brøndal. Dans l'analyse de termes sémantiques à plusieurs composantes, il reconnaît la dominance de l'un de ces effets de sens par rapport aux autres, lesquels demeurent présents. Dans la Centrale Montemartini, le musée archéologique est aujourd'hui dominant par rapport au musée technique. Ce fait est en partie induit par la communication faite à l'extérieur : les musées capilotins font savoir qu'une partie de leurs collections est exposée à la Centrale. Ce faisant, ils invitent à la visite. L'amateur d'antiquité qui se déplace ainsi le fait dans un but prédéterminé, ce qui produit la dominance du musée archéologique sur le musée technique. Si demain le quartier de la via Ostiense était remodelé, avec le gazomètre et les marchés généraux, il se pourrait que ce quartier devienne un pôle de l'archéologie industrielle. Une communication bien faite pourrait attirer là des visiteurs intéressés par le musée technique. Alors, il serait possible que la lecture du musée technique devienne dominante par rapport à celle du musée archéologique. Il reste que l'aménagement actuel de ce musée est plus orienté vers la mise en valeur des objets archéologiques.

\section{La structure hiérarchique du discours muséal}

Au terme du parcours analytique, il convient d'opérer un retour sur les procédés mis en œuvre afin d'en expliciter les mécanismes et d'en tirer des conclusions. Nous avons distingué, avec récurrence et à propos de chaque salle, trois niveaux dans l'analyse : objets, aménagement et architecture. Reprenons chacune de ces catégories afin d'en examiner les propriétés générales et de préciser les relations qui les relient en tant que catégories.

\section{La catégorie des objets muséaux}

Elle comprend des objets du monde naturel, tels que des sculptures et des machines. Envisagés en tant qu'entités porteuses de sens, ces objets sont insérés dans une chaîne communicative placée entre un énonciateur et un énonciataire. Dès lors, il est légitime de projeter sur eux le modèle discursif aux fins de l'analyse. Interrogés en tant que discours non verbal, les objets livrent du sens. De ce point de vue, ce sont des sujets délégués qui portent des messages non verbaux inscrits par des sujets reconstruits par présupposition. Dans une mesure variable, le sujet énonciateur de l'objet y a laissé ses marques, qui sont autant d'éléments d'énonciation inscrits dans l'énoncé-objet. L'histoire de certains objets muséaux manifeste des étapes caractéristiques récurrentes constituant ce que nous avons appelé le parcours narratif de l'objet (création, mise en œuvre, trouvaille, reconnaissance, restauration, installation au musée). Chacune de ces étapes modifie le contenu sémantique de l'objet, ce qui caractérise le contenu final comme un sens stratifié.

Le sens de chaque objet est modifié par le simple fait d'être intégré dans la collection d'un musée. Car celle-ci présuppose des critères de sélection. À l'instar des objets fragmentés déterrés par les fouilles, les collections sont constituées par les fragments que sont les objets mêmes, et toute collection est incomplète a priori : une nouvelle découverte archéologique peut venir modifier la composition de la collection et le sens des objets réunis. Étant porteuse de sens, la collection est assimilable à un discours dont l'énonciateur est le collectionneur : en collectant, celui-ci cherche à compléter le sens. La disposition des objets de la collection par groupes de voisinage, par éloignements, par alignements ou par coordination des orientations... fait apparaître des effets de sens particuliers sur des parties de la collection. Ces opérations constituent autant d'actes énonciatifs organisant des énoncés spatiaux.

Ces faits font apparaître deux niveaux d'organisation de l'expression et du contenu : celui des objets isolés, celui des groupes d'objets. Les groupes révèlent l'existence d'un sujet énonciateur muséal, inscrit dans la collection et non présent dans l'objet individuel. Ce n'est pas le sujet de discours présupposé par l'objet mais un nouveau sujet prenant en charge, via la collection, l'objet et son discours.

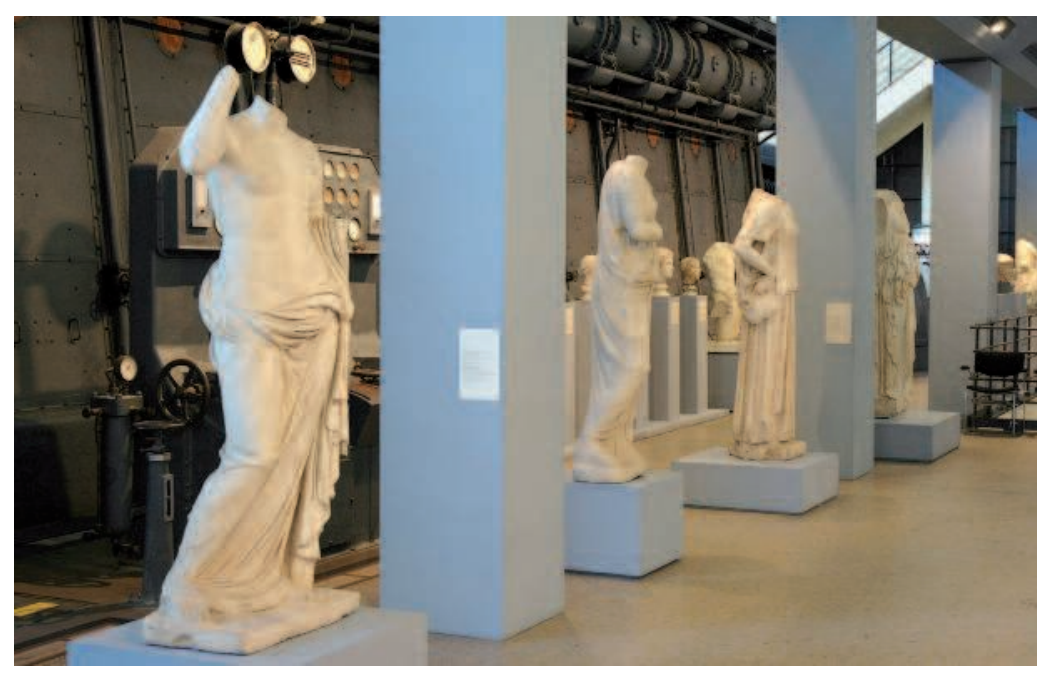

Alignements de sculptures dans la Salle des Machines (c) M. Hammad 


\section{La catégorie de l'aménagement muséal}

Il n'y a d'aménagement muséal qu'en fonction des objets exposés : l'éclairage n'a de sens que par rapport à ce qu'il met en lumière, les appuis n'ont de sens que par rapport aux objets qu'ils supportent. Par conséquent, la catégorie de l'aménagement présuppose la catégorie des objets.

Dans la mesure où les aménagements sont destinés à assurer au visiteur de meilleures conditions du regard, la catégorie de l'aménagement véhicule des investissements modaux constitutifs de la compétence du sujet spectateur. Dans la mesure où l'aménagement assure la séparation entre les messages délivrés par des objets voisins, lesquels peuvent être perturbateurs l'un pour l'autre ce qui les ferait apparaître comme des anti-sujets l'un pour l'autre, l'aménagement véhicule des investissements modaux constitutifs de la compétence de l'objet muséal. Dans la mesure où de telles procédures permettent de distinguer un objet de l'autre, elles sont énonciatives, ancrant les objets dans des espaces de référence différents. En fournissant aux objets un cadre spatial qui leur est assigné, l'aménagement en détermine l'expressivité et l'importance relative. Bref, l'aménagement détermine partiellement l'expression et le contenu des objets exposés.

Dans la Centrale Montemartini, certaines des machines exposées jouent deux rôles actantiels à la fois : elles sont des objets exposés au regard des visiteurs, elles font partie du dispositif de l'aménagement, servant d'appui rétrospectif à un certain nombre de sculptures antiques.

Étant porteur d'effets de sens, l'aménagement est l'expression d'un niveau discursif qui présuppose son propre énonciateur. En l'occurrence, il s'agit de l'acteur collectif constitué par les autorités muséales (conservateurs, architectes, entreprises). Dès lors, l'aménagement apparaît comme un sujet délégué statique, mis en place par l'énonciateur muséal.

\section{La catégorie de l'architecture}

Au musée, la catégorie de l'architecture présuppose celle des objets qu'elle abrite et offre aux regards. Parallèlement, l'architecture est apparue régir la disposition et des objets et de l'aménagement. Elle est donc investie de valeurs modales rendant certaines actions possibles, interdisant d'autres actions. Étant parfois un objet à voir pour ses qualités propres, l'architecture est assimilable aux objets muséaux. En de tels cas, elle est dotée d'un rôle actantiel double : elle est à la fois objet à voir et dispositif régissant les objets.

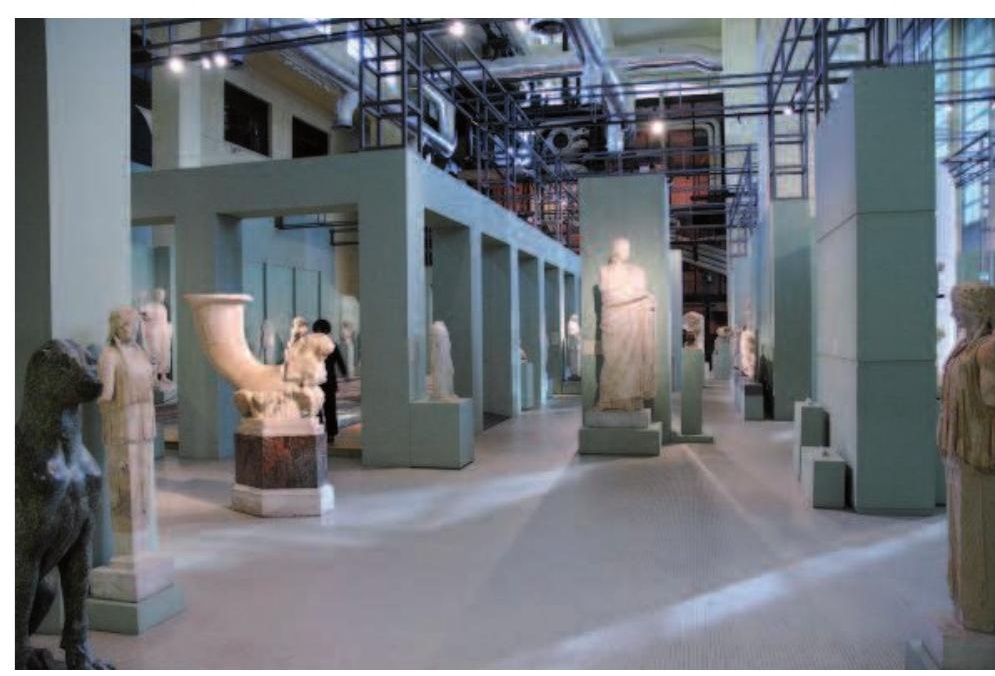

L'aménagement muséographique de la Salle des Chaudières (c) M. Hammad

Dans la mesure où elle est porteuse de sens, l'architecture constitue un niveau discursif en soi, présupposant un énonciateur. Cette position syntaxique peut, selon les cas, être remplie par l'acteur collectif « conservation du musée » ou relever, comme dans le cas de la centrale électrique ou des palais réutilisés, d'instances plus complexes possédant leur propre histoire et juxtaposant leur discours à celui du musée.

\section{La relation d'enchâssement discursif}

Au plan de l'expression, les objets du musée sont enchâssés dans le dispositif de l'aménagement, lequel est enchâssé dans l'architecture. Trois relations de présupposition ont été relevées entre ces termes : l'architecture présuppose les objets, l'architecture présuppose l'aménagement, l'aménagement présuppose les objets.

Au plan du contenu, nous avons relevé trois niveaux discursifs liés aux catégories citées, et entretenant des relations de contrôle : le discours de l'aménagement contrôle et régit le discours des objets, le discours architectural contrôle et régit le discours de l'aménagement, le discours architectural contrôle et régit le discours des objets. Les trois niveaux discursifs coexistent simultanément au sein du musée et contribuent à en articuler le discours syncrétique.

\section{La hiérarchie des niveaux linguistiques}

Greimas (3) reprend à Hjelmslev la distinction, dans tout discours, de niveaux hiérarchiques logiquement dépendants : le langage objet est présupposé par le métalangage descriptif, lequel est présupposé par le 
métalangage méthodologique qui le régule, lequel est présupposé par le métalangage épistémologique qui le régule. Ce dispositif est isomorphe à l'articulation des types logiques posée par Russell (4) pour donner une solution aux paradoxes connus depuis l'Antiquité sous la forme dite d'Epiménide le menteur. En toute rigueur, la chaîne des niveaux logiques n'est pas limitée. Cependant, Hjelmslev et Greimas constatent que trois niveaux hiérarchiques suffisent en général, le troisième niveau (épistémologique) étant à toutes fins utiles pris pour l'ensemble des niveaux supérieurs aux deux premiers.

La relation de rection relevée entre les catégories de notre analyse architecturale assure le contrôle de l'un des niveaux par l'autre. Elle est donc logiquement isomorphe à celle reconnue par Greimas, Hjelmslev, Russell et Gödel. Elle n'en est que la forme manifestée en sémiotique de l'espace. Elle illustre le fait que la relation logique redouble, dans les conditions de cette analyse, la relation de présupposition entre énoncé et énonciation. En d'autres termes, la relation entre les catégories des objets muséaux, de l'aménagement muséal et de l'architecture muséale est métalinguistique, régissante, énonciative ${ }^{(5)}$. Les niveaux supérieurs prennent en charge les niveaux inférieurs et les surdéterminent.

Ces remarques formelles inscrivent le discours muséal dans un contexte plus large, celui des discours logiques et scientifiques visant la production d'un savoir contrôlé. Ce qui le rend comparable à ces derniers, tout en maintenant les caractères qui l'en distinguent, en particulier son expression syncrétique et non verbale.

\section{L'interprétation du discours muséal}

\section{Lecture et déchiffrement}

La lecture sémiotique de la Centrale Montemartini n'est pas entrée dans le détail des procédures de mise en correspondance du contenu avec l'expression. Nous avons explicité les contenus que nous percevions en nous référant à notre apprentissage antérieur de l'architecture, de l'histoire de l'art et des arts plastiques. Pour l'identification des œuvres et de leur parcours narratif, nous avons exploité les cartels apposés près des œuvres, les complétant par le catalogue et par des publications archéologiques diverses. On reconnaîtra dans ce court rappel trois composants :

- les objets muséaux mis en espace, l'ensemble ayant une expression non verbale (a),

- une documentation verbale relative à ces objets, organisée en trois ensembles de plus en plus étendus : les cartels, le catalogue, des ouvrages archéologiques (b), - une formation antérieure multiforme du lecteur (c).
D'un tel point de vue, l'ensemble (b) apparaît comme un complément spécifique à la compétence (c), relatif à l'ensemble (a). On y trouve des informations que le dispositif non verbal peut difficilement transmettre, telles que les dates du parcours narratif de l'objet, les personnages, les lieux et les événements historiques avec lesquels l'objet a été en relation. Or le savoir consigné dans ces documents provient d'éléments non verbaux transcrits et/ou explicités verbalement. Pour s'en convaincre, il suffit de penser à l'information topographique nécessaire : le pays et les sites sont des éléments non verbaux que les géographes s'échinent à décrire dans des textes. L'histoire est faite d'événements relatés dans des textes. L'histoire de l'art décrit des objets matériels, comme l'histoire de l'architecture. Bref, la matière première de notre savoir livresque provient du monde non verbal qui nous entoure. Le discours scientifique ajoute à la description dudit monde les niveaux méthodologiques et épistémologiques construits afin de contrôler cette dernière. Il n'y a pas une différence de nature entre le savoir tiré de l'ensemble non verbal (a) et le savoir tiré de l'ensemble verbal (b). Au plan du contenu, ils se rejoignent, ils ne diffèrent que par l'expression.

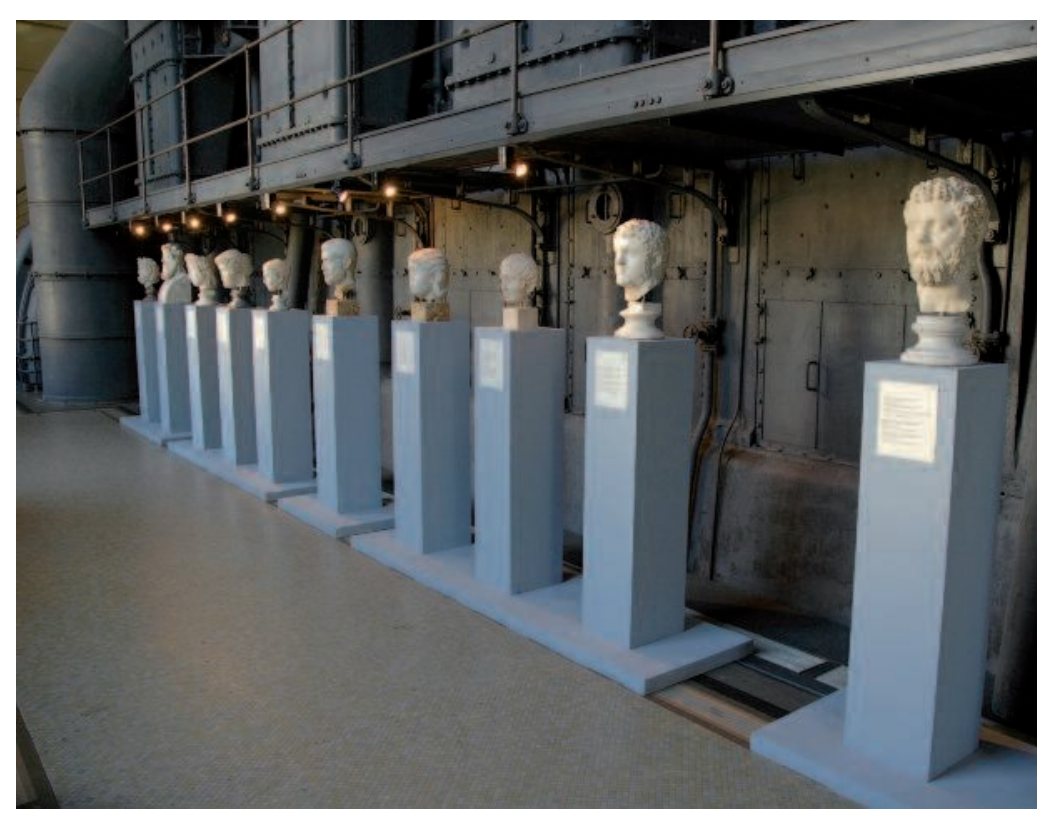

L'allée latérale de la Salle des Machines avec des sculptures alignées devant un moteur. (c) M. Hammad 
Le sujet du déchiffrement

Les visiteurs ne sont pas dotés du même bagage antérieur, comme ils n'ont pas accès à la même documentation. Il en découle qu'il y a autant de lectures que de visiteurs. La lecture que nous avons menée est celle d'un architecte amateur d'antiquités et préoccupé par le sens. D'autres lectures sont possibles.

Le lecteur du musée ne se réduit pas à un sujet cognitif : c'est aussi un sujet pragmatique qui parcourt les salles afin d'en regarder les objets. Étant donné que l'extension des lieux et leur topologie autorisent de multiples circuits, il y a autant de parcours linéaires générateurs d'effets de sens. Nous n'avons pas abordé ces variantes de lecture qui introduisent une variabilité du résultat. L'étude des manières de visiter montre que le comportement des sujets dans l'espace muséal dépend de leur compétence interprétative : on reconnaît le parcours des experts qui, ayant sélectionné quelques objets à l'avance, se déplacent rapidement de l'un à l'autre, restant longtemps devant chaque pièce sélectionnée. À cette manière s'oppose tant le parcours aléatoire de visiteurs qui n'y connaissent rien, que le parcours systématique des visiteurs qui se forment et s'arrêtent devant toutes les pièces. Ces variantes de la visite sont dessinées sur la trame constante du dispositif muséal mis en place par les conservateurs. Organisant des trajets, invitant au mouvement, indiquant les œuvres par des lumières directionnelles, le dispositif muséal met en place les composantes de la compétence du sujet visiteur. Dans la mesure où ce dispositif est inévitable, tous les visiteurs du musée sont manipulés dans leur parcours pragmatique.

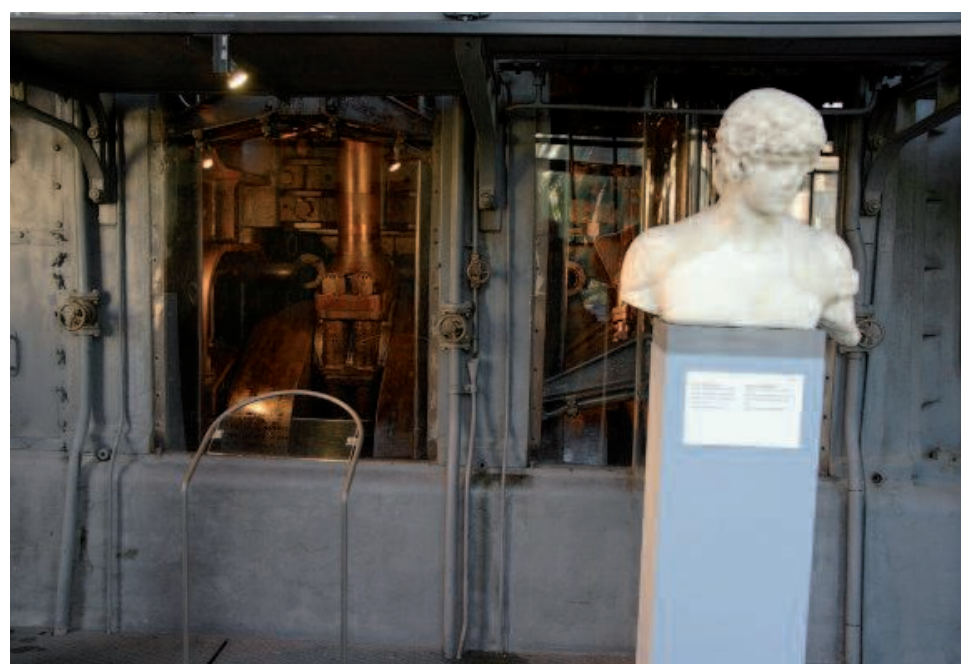

Dans la Salle des Machines, entre deux bustes de marbre, un panneau transparent laisse entrevoir une bielle. (c) M. Hammad
Enfin, le visiteur de musée ne voit pas que des objets muséaux, il voit aussi d'autres visiteurs. Ces derniers se déplacent. Leur nombre peut constituer une gêne à la visite, dans la mesure où certains corps s'interposent entre le spectateur et l'objet qu'il voudrait regarder. Le rythme de visite des uns et des autres peut varier, constituant une gêne d'ordre pragmatique et non cognitif. Dans tous ces cas, le visiteur joue deux rôles actantiels, étant à la fois le sujet observateur et le sujet impliqué. Ce qui ouvre d'autres pistes à l'analyse de la lecture.

La Centrale Montemartini juxtapose deux discours muséaux parallèles relativement étrangers l'un à l'autre. Indépendamment de ses qualités plastiques, ce fait s'est révélé fort intéressant à l'analyse. Il est apparu aussi que la muséographie de la partie antique est novatrice, cohérente et élaborée. À ce titre, elle mérite d'être louée. D'un point de vue sémiotique, elle a imposé d'examiner de plus près l'histoire des objets muséaux, ce qui a fait reconnaître un parcours narratif pour chacun d'eux. La disposition des objets dans les salles a fait reconnaître l'une des finalités peu explicites des musées d'archéologie, celle de contribuer à reconstituer les cultures disparues. L'analyse d'un discours muséal à deux composantes parallèles a imposé de réfléchir sur les transformations sémantiques réalisées par tout musée lors de la mise en exposition de ses objets. Enfin, la reconnaissance de la structure hiérarchique du discours muséal a permis de le rapprocher de l'ensemble des discours scientifiques. Ce qui fait avancer la sémiotique des musées en général.

\section{Notes}

(1) Cette étude résume l'un des sujets traités lors d’un séminaire doctoral dispensé à l'université de Roma-La Sapienza sur le thème de la réutilisation des bâtiments anciens. Une version détaillée de l'analyse a été publiée en 2006 chez Meltemi à Rome dans un ouvrage collectif dirigé par I. Pezzini et P.-L. Cerveli, intitulé Scene del consumo : dallo shopping al museo.

(2) Les fouilles ont retrouvé, sur les collines du Palatin et du Capitole, des traces de l'Âge du Bronze et de l'Âge du Fer.

(3) Greimas, A.-J. Sémantique structurale. Paris : Larousse, 1966.

(4) Russell, B. La théorie des types logiques, La revue de métaphysique et de morale, vol. XVIII, 1910. Article reproduit en 1969 dans Cahiers pour l'analyse, $\mathrm{n}^{\circ} 10$, Seuil.

(5) Hammad, M. L'énonciation, procès et système, in Langages 70, Larousse, 1983 